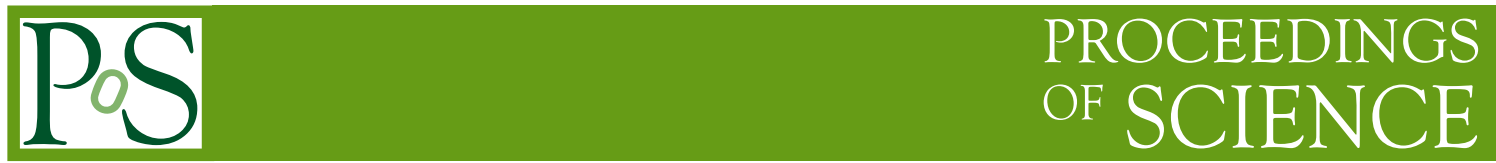

\title{
The static three-quark potential of various quark configurations
}

\author{
Miho Koma* \\ Nihon University, College of International relations \\ E-mail: koma.mihoenihon-u.ac.jp \\ Yoshiaki Koma ${ }^{\dagger}$ \\ National Institute of Technology, Numazu College \\ E-mail: komadnumazu-ct.ac.jp
}

We study the static three-quark potential in SU(3) lattice gauge theory at zero temperature from the Polyakov loop correlation function. By employing the multilevel algorithm, we obtain the potential for $O(200)$ sets of three-quark configurations of various sizes and geometries with less statistical and systematic errors, where the three quarks are put at the vertices of the acute, right, obtuse triangles, and are also put in line. We investigate the possible functional form of the threequark potential.

The 33rd International Symposium on Lattice Field Theory

$14-18$ July 2015

Kobe International Conference Center, Kobe, Japan

*Speaker.

†.K. is partially supported by JSPS KAKENHI Grant Number 24740176. The main computation was performed on the NEC SX8 at Research Center for Nuclear Physics (RCNP), Osaka University, Japan. 


\section{Introduction}

The static three-quark potential in QCD is expected to play an important role not only in heavy baryon spectroscopy but also in clarifying the confining structure of the three-quark system. Lattice QCD simulations offer nonperturbative computation of the interquark potential, and the

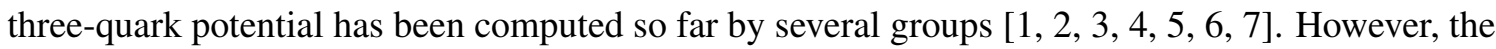
determination of the functional form of the potential is not straightforward compared to that of the quark-antiquark potential, since the functional form is of course dependent on how the color flux tube is formed among the three quarks, which is not known a priori due to nonperturbative nature of the QCD vacuum.

Two types of the functional form deduced from the $\Delta$-shaped and the $Y$-shaped flux-tube pictures have mainly been tested in earlier studies, where the three quarks are assumed to be located at the edge of these characters. The functional form of the potential of these two descriptions is unfortunately not so different with each other in practice, so that the precise lattice data with less statistical and systematic errors are clearly required before evaluating the goodness of the functional form. In all the previous studies, the three-quark Wilson loop as schematically shown in Fig. W (left) is computed, which is a natural extension of the ordinary rectangular Wilson loop for the quark-antiquark system. However, the later study on the flux-tube profile in the three-quark system with the three-quark Wilson loop shows that the profile can be affected by the shape of the spatial Wilson lines [ $[\mathrm{d}]$. The numerical procedure for computing the potential and the profile is not exactly the same, but it is certainly preferable to obtain the potential data from the three-quark sources without the spatial Wilson lines if the functional form is of interest.

In this work we compute the three-quark potential of various quark configurations from the Polyakov loop correlation function (PLCF) at zero temperature. Our result is free from the systematic effect due to the spatial Wilson lines, since the PLCF is consisted only of the time-like Polyakov loops as illustrated in Fig. W(right). A problem may be the smallness of the expectation value of the PLCF at long distances at zero temperature, which means that the ordinary simulations are ineffective as the signal is easily obscured by the statistical noise. This problem can, however, be solved by employing the multilevel algorithm [可]. Remarkably, we find that this algorithm also allows us to compute the PLCF only from one gauge configuration by tuning the simulation parameters [ए]]. We investigate $O(200)$ sets of the three-quark configurations of various sizes and
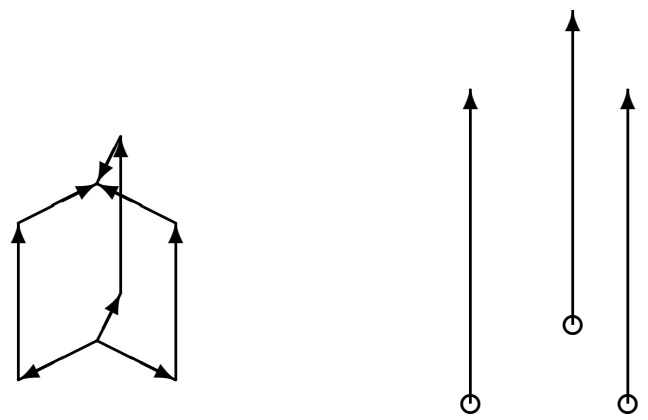

Figure 1: The three-quark Wilson loop (left) and the Polyakov loop correlation function (PLCF) for the three-quark system (right). 
geometries, including some extreme cases that the three quarks are put at the vertex of the obtuse triangle whose maximum inner angle is greater than 120 degrees and are also put in line.

We find that most of the three-quark potential from the triangle configurations that the maximum inner angle is smaller than 120 degrees can fall into one curve. This result supports a picture that the flux tube is formed basically so as to minimize the total length of lines connecting the three quarks. We compare the three-quark potential to the sum of the quark-antiquark potential and find a systematic deviation, which indicates that the two-body interaction is not enough to describe the three-quark system.

\section{Numerical procedures}

We perform simulations of SU(3) lattice gauge theory in four dimensions with the lattice volume $24^{4}$, where periodic boundary conditions are imposed in all space-time directions. We use the standard Wilson gauge action at $\beta=6.00$ and the corresponding lattice spacing is $a=$ $0.093[\mathrm{fm}][\mathrm{W}]$. One Monte Carlo update consists of one heatbath, followed by five overrelaxation steps.

We compute the PLCF composed of three Polyakov loops, $\operatorname{Tr} P\left(\vec{x}_{1}\right) \operatorname{Tr} P\left(\vec{x}_{2}\right) \operatorname{Tr} P\left(\vec{x}_{3}\right)$, where the spatial location of each Polyakov loop, $\vec{x}_{1}, \vec{x}_{2}, \vec{x}_{3}$ corresponds to that of a quark. We then put the three Polyakov loops in three-dimensional space as shown in Fig. \, where various three-quark configurations can be classified into four types such that the three quarks are put at the vertices of the acute, right, obtuse triangles, and are also put in line. Note that the acute triangles contain regular and isosceles triangles, and the obtuse triangles are further classified into two types by the maximum inner angles $\theta_{\max }$, greater or less than 120 degrees (see Table W). Based on the law of cosine, $\theta_{\max }$ can be written in terms of interquark distance $r_{1}=\left|\vec{x}_{2}-\vec{x}_{3}\right|, r_{2}=\left|\vec{x}_{3}-\vec{x}_{1}\right|, r_{3}=\left|\vec{x}_{1}-\vec{x}_{2}\right|$ as

$$
\cos \theta_{\max }=\frac{r_{\max }\left(r_{1}^{2}+r_{2}^{2}+r_{3}^{2}-2 r_{\max }^{2}\right)}{2 r_{1} r_{2} r_{3}}
$$

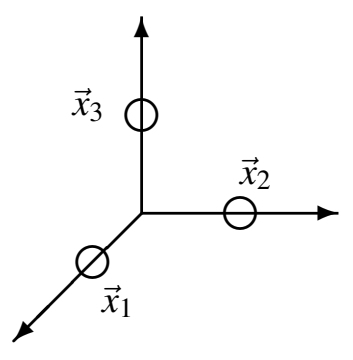

(i) acute

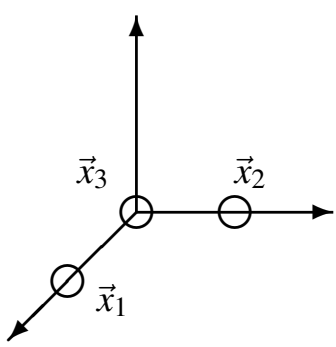

(ii) right

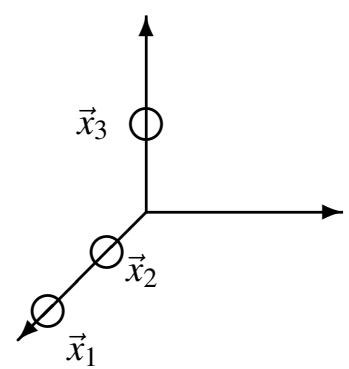

(iii) obtuse

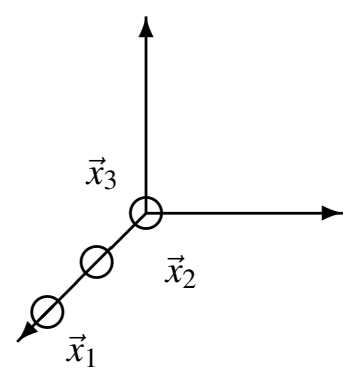

(iv) line

Figure 2: The spatial location of the Polyakov loops, where the three quarks are put at the vertices of the acute (i), right (ii), obtuse (iii) triangles, and are also put in line (iv). 


\begin{tabular}{|c|c|c|c|c|}
\hline \multicolumn{2}{|c|}{ classification } & \multicolumn{3}{|c|}{ The number of configurations } \\
\hline \multirow[t]{2}{*}{ acute } & isosceles (regular) & $65(7)$ & 135 & \multirow{6}{*}{218} \\
\hline & right & 43 & (of which 87 satisfy $r_{\min }>2 a$ ) & \\
\hline \multirow[t]{2}{*}{ obtuse } & $\theta_{\max }<120^{\circ}$ & 27 & $\bullet$ & \\
\hline & $\theta_{\max } \geq 120^{\circ}$ & 28 & (of which 3 satisfy $r_{\min }>2 a$ ) & \\
\hline \multicolumn{2}{|r|}{ line } & 32 & (of which 12 satisfy $r_{\min }>2 a$ ) $\Delta$ & \\
\hline \multicolumn{2}{|c|}{ q-qq (quark-diquark) } & 23 & 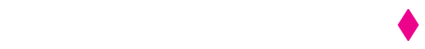 & \\
\hline
\end{tabular}

Table 1: The classification of the $O(200)$ three-quark configurations investigated in our simulation in terms of the triangle shape formed by the three quarks, where $r_{\min }=\min \left(r_{1}, r_{2}, r_{3}\right)$ denotes the minimum interquark distance. The symbols correspond to that used in Fig. 田.
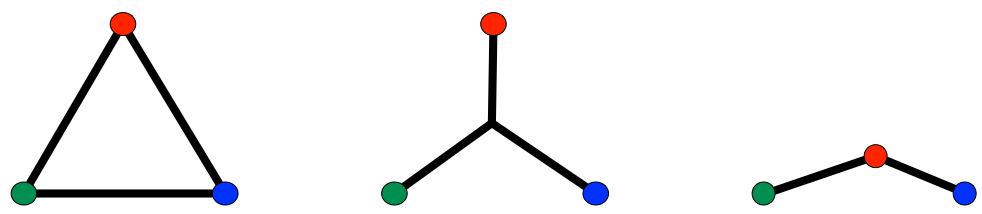

Figure 3: The $\Delta$ - (left), $Y$ - (middle) and $\Lambda$ - (right) shaped flux tubes, where colored circles denote the quarks. The length of the thick lines in each flux tubes corresponds to the $\Delta, Y$ and $\Lambda$ distances defined in Eqs. (B.D), (B.2.) and (B.3)), respectively.

where $r_{\max }=\max \left(r_{1}, r_{2}, r_{3}\right)$ denotes the maximum interquark distance. The three-quark potential $V_{3 q}$ is then extracted from the expectation value of the PLCF by

$$
V_{3 \mathrm{q}}\left(\vec{x}_{1}, \vec{x}_{2}, \vec{x}_{3}\right)=-\frac{1}{T} \ln \left\langle\operatorname{Tr} P\left(\vec{x}_{1}\right) \operatorname{Tr} P\left(\vec{x}_{2}\right) \operatorname{Tr} P\left(\vec{x}_{3}\right)\right\rangle+O\left(\frac{1}{T} e^{-(\Delta E) T}\right),
$$

where the contribution from the excited states $O\left(\frac{1}{T} e^{-(\Delta E) T}\right)$ are negligible at zero temperature as $T=24 a$ is already large enough.

We employ the multilevel algorithm to evaluate the expectation value of the PLCF. The idea of the algorithm is to compute a desired correlation function, which may have an extremely small expectation value, from the product of relatively large sublattice averages of its components, where the sublattices are defined by dividing the lattice volume into several layers along the time direction. During the computation of the sublattice averages, the spatial links at the sublattice boundaries are fixed. We denote the number of the sublattice $N_{\text {sub }}$ and the number of internal update for the sublattice average $N_{\text {iupd }}$. A detailed description of the method for the PLCF in the three-quark system is given in Ref. [ए0]. We use the same parameter set such as $N_{\text {sub }}=6$ and $N_{\text {iupd }}=500000$ used in Ref. [एँ]], which enables us to compute the three-quark potential from one gauge configuration.

\section{Numerical results}

We introduce various distances called $\Delta, Y, \Lambda$ as schematically shown in Fig. [3, which correspond to the length of the possibly-formed flux tubes among the three quarks. By using the 


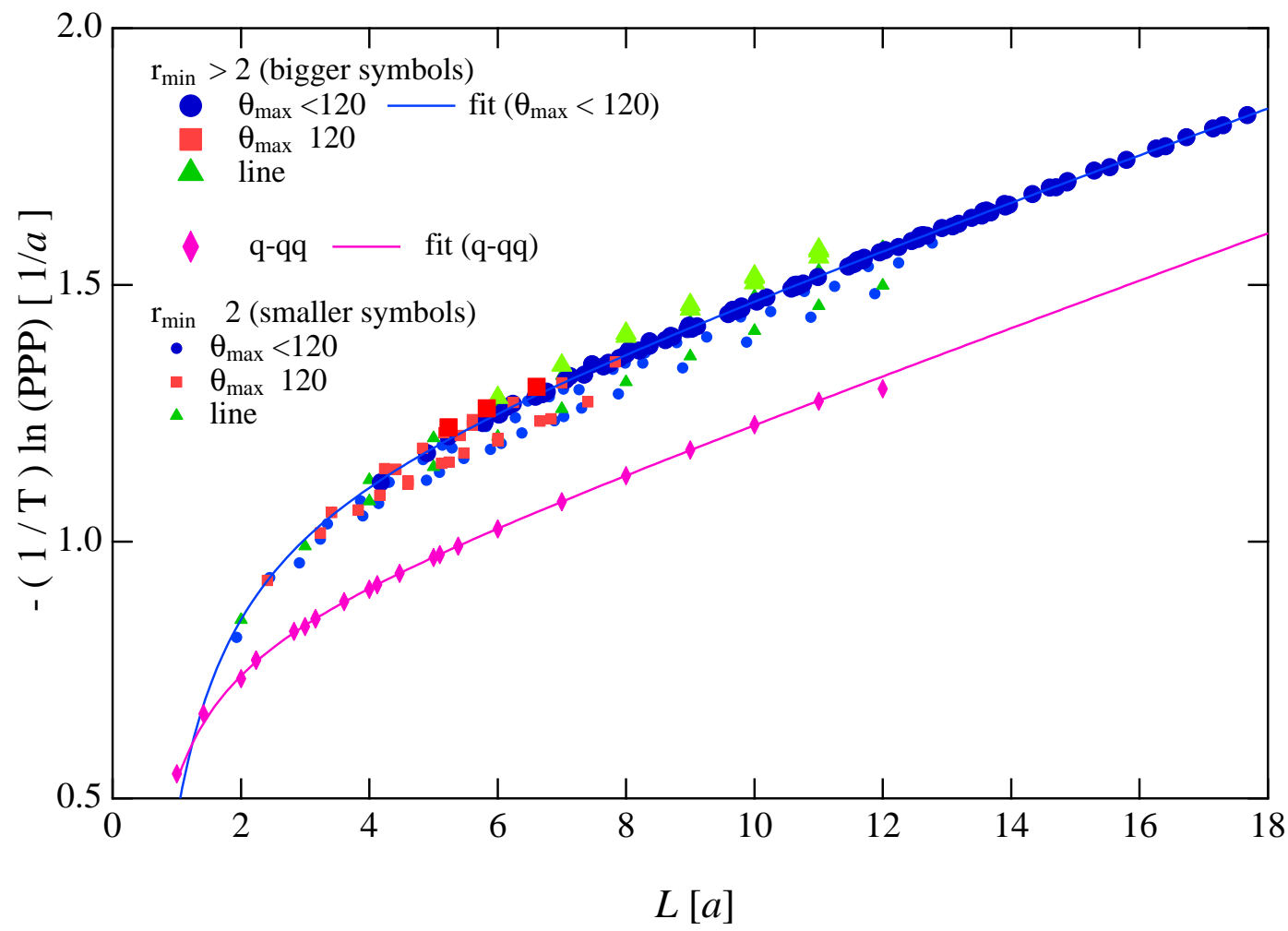

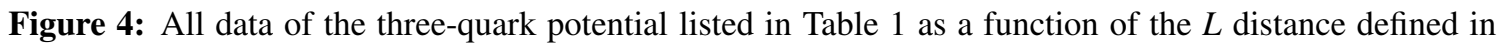
Eq.(B.4) in units of lattice spacing $a$. The bigger symbols denote the potential from the configuration for $r_{\min }>2 a$, while the smaller symbols for $r_{\min } \leq 2 a$.

interquark distances $r_{1}, r_{2}, r_{3}$, these distances are written as

$$
\begin{aligned}
& \Delta=r_{1}+r_{2}+r_{3} \\
& Y=\sqrt{\frac{\left(r_{1}^{2}+r_{2}^{2}+r_{3}^{2}\right)+\sqrt{3\left(r_{1}+r_{2}+r_{3}\right)\left(-r_{1}+r_{2}+r_{3}\right)\left(r_{1}-r_{2}+r_{3}\right)\left(r_{1}+r_{2}-r_{3}\right)}}{2}}, \\
& \Lambda=\Delta-r_{\max } .
\end{aligned}
$$

We also introduce the minimal total length of the flux tube $L$, which is given by the $Y$ or the $\Lambda$ distance depending on the $\theta_{\max }$ defined in Eq. (2.]), as

$$
L=\left\{\begin{array}{l}
Y\left(\theta_{\max }<120^{\circ}\right) \\
\Lambda\left(\theta_{\max } \geqq 120^{\circ}\right)
\end{array} .\right.
$$

In Fig. $⿴$, we plot all data of the three-quark potential as a function of the $L$ distance. By fitting the data denoted by big-blue circles (87 points), which belong to the configurations for $r_{\min }=\min \left(r_{1}, r_{2}, r_{3}\right)>2 a$ and $\theta_{\max }<120^{\circ}$, to the conventional functional form,

$$
V_{\text {fit }}(L)=-\frac{A}{L}+\sigma L+\mu
$$



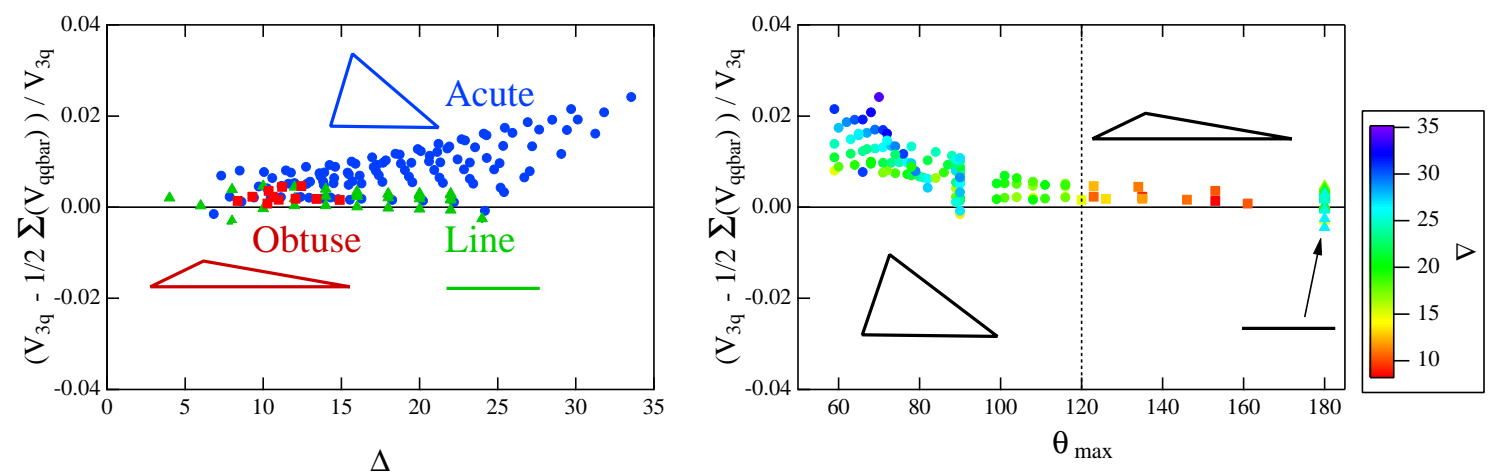

Figure 5: A relative deviation of the three-quark potential from the sum of the three quark-antiquark potential as a function of the $\Delta$ distance (left) and of the $\theta_{\max }$ (right).

we find that they fall into one curve with the parameters as $A_{3 q}=0.68(3), \sigma_{3 q}=0.0433(3), \mu_{3 q}=$ 1.102(6). In this case the $L$ distance is nothing but the $Y$ distance. The potential of the configurations for $r_{\min } \leq 2 a$ are not described by the same fitting parameters, which may be due to the cutoff effect. In Fig. 团, we also plot the quark-diquark potential. By fitting these data to the same functional form as in Eq.(B.5), we obtain $A_{\mathrm{q}-\mathrm{qq}}=0.320(8), \sigma_{\mathrm{q}-\mathrm{qq}}=0.0450(4), \mu_{\mathrm{q}-\mathrm{qq}}=1.102(6)$, where the data $2 a \leq L \leq 11 a$ are taken into account (20 points).

We then compare the three-quark potential $V_{3 q}$ to the half of the sum of the three quarkantiquark potentials,

$$
\frac{1}{2} \sum V_{\mathrm{q} \overline{\mathrm{q}}}(\Delta)=\frac{1}{2}\left(V_{\mathrm{q} \overline{\mathrm{q}}}\left(r_{1}\right)+V_{\mathrm{q} \overline{\mathrm{q}}}\left(r_{2}\right)+V_{\mathrm{q} \overline{\mathrm{q}}}\left(r_{3}\right)\right)
$$

where we use

$$
V_{\mathrm{q} \overline{\mathrm{q}}}(r)=-\frac{0.296}{r}+0.0463 r+0.749
$$

for the quark-antiquark potential, which was obtained from the use of the multilevel algorithm with the same simulation parameters [[2]. In Fig. [1, we plot relative deviation of $V_{3 \mathrm{q}}$ from $\frac{1}{2} \sum V_{\mathrm{q}}$, namely $\left(V_{3 \mathrm{q}}-\frac{1}{2} \sum V_{\mathrm{q} \overline{\mathrm{q}}}(\Delta)\right) / V_{3 \mathrm{q}}$, as a function of the $\Delta$ distance and the $\theta_{\max }$, respectively. We find a systematic deviation from the zero line, which tends to increase up to three percent as the $\Delta$ distance becomes large and the $\theta_{\max }$ approaches 60 degrees. This means that the sum of the quark-antiquark potential cannot describe the full behavior of the three-quark potential. On the other hand, the deviation is less than one percent for the obtuse-triangle configurations with $\theta_{\max }>120^{\circ}$ and the line configurations.

\section{Summary}

We have computed the static three-quark potential from the PLCF at zero temperature, which is free from the systematic effect due to the spatial Wilson lines as in the three-quark Wilson loop. By virtue of the multilevel algorithm, we have obtained clean potential data from one gauge configuration, where the maximum $\Delta$ distance reaches about $3.3 \mathrm{fm}$. We have investigated $O(200)$ 
sets of three-quark configurations of various sizes and geometries, where the three quarks are put at the vertices of the acute, right, obtuse triangles, and are also put in line.

We have tested various functional forms parametrized by the $\Delta, Y, \Lambda$ distances. We have found that the potential data for $r_{\min }>2 a$ and $\theta_{\max }<120^{\circ}$ can fall into one curve with the $Y$ distance. This result supports a picture that the flux tube is formed basically so as to minimize the total length of lines connecting the three quarks. In order to find the functional form for the rest of the potential data, it is quite important to discriminate the short distance effect due to finite lattice spacing.

We have examined systematically whether the three-quark potential can be described by the sum of the quark-antiquark potential. We have found a systematic deviation especially for acutetriangle configurations, which tends to increase up to three percent as the $\Delta$ distance increases and the $\theta_{\max }$ approaches 60 degrees. This result infers that the force beyond the two-body one is certainly present, which shows up when the three quarks are extended large enough to form a junction of the flux tube inside the triangle.

\section{References}

[1] R. Sommer and J. Wosiek, Baryonic loops and the confinement in the three quark channel, Phys.Lett. B149 (1984) 497.

[2] R. Sommer and J. Wosiek, Baryonic strings on a lattice, Nucl.Phys. B267 (1986) 531.

[3] H. Thacker, E. Eichten, and J. Sexton, The three-body potential for heavy quark baryons in lattice QCD, Nucl.Phys.Proc.Suppl. 4 (1988) 234.

[4] G. S. Bali, QCD forces and heavy quark bound states, Phys.Rept. 343 (2001) 1-136, [hep-ph/0001312].

[5] T. T. Takahashi, H. Matsufuru, Y. Nemoto, and H. Suganuma, The three quark potential in the $S U(3)$ lattice QCD, Phys.Rev.Lett. 86 (2001) 18-21, [hep-1at/0006005].

[6] T. T. Takahashi, H. Suganuma, Y. Nemoto, and H. Matsufuru, Detailed analysis of the three quark potential in SU(3) lattice QCD, Phys.Rev. D65 (2002) 114509, [hep-1at/0204011].

[7] C. Alexandrou, P. De Forcrand, and A. Tsapalis, The static three quark SU(3) and four quark SU(4) potentials, Phys.Rev. D65 (2002) 054503, [hep-1at/0107006].

[8] F. Bissey, F.-G. Cao, A. Kitson, A. Signal, D. Leinweber, et al., Gluon flux-tube distribution and linear confinement in baryons, Phys.Rev. D76 (2007) 114512, [hep-1at/0606016].

[9] M. Lüscher and P. Weisz, Locality and exponential error reduction in numerical lattice gauge theory, JHEP 09 (2001) 010, [hep-1at/0108014].

[10] Y. Koma and M. Koma, The static three-quark potential from the Polyakov loop correlation function, PoS LATTICE2014 (2014) 352.

[11] S. Necco and R. Sommer, The $N(f)=0$ heavy quark potential from short to intermediate distances, Nucl. Phys. B622 (2002) 328-346, [hep-1at/0108008].

[12] Y. Koma and M. Koma, The static potential from the selected intermediate states of gluons, PoS LATTICE2013 (2013) 469. 